\title{
Exploring the role of X-ray reprocessing and irradiation in the anomalous bright optical outbursts of A0538-66
}

\author{
L. Ducci ${ }^{1,2}$, S. Mereghetti ${ }^{3}$, K. Hryniewicz ${ }^{4}$, A. Santangelo ${ }^{1}$, and P. Romano ${ }^{5}$ \\ ${ }^{1}$ Institut für Astronomie und Astrophysik, Kepler Center for Astro and Particle Physics, Eberhard Karls Universität, Sand 1, \\ 72076 Tübingen, Germany \\ e-mail: ducci@astro.uni-tuebingen.de \\ 2 ISDC Data Center for Astrophysics, Université de Genève, 16 Chemin d’Écogia, 1290 Versoix, Switzerland \\ 3 INAF - Istituto di Astrofisica Spaziale e Fisica Cosmica, Via E. Bassini 15, 20133 Milano, Italy \\ 4 Nicolaus Copernicus Astronomical Center, Polish Academy of Sciences, Bartycka 18 00-716 Warsaw, Poland \\ 5 INAF - Osservatorio Astronomico di Brera, Via Bianchi 46, 23807 Merate, LC, Italy
}

Received 5 October 2018 / Accepted 13 February 2019

\begin{abstract}
Context. In 1981, the Be/X-ray binary A0538-66 showed outbursts characterized by high peak luminosities in the X-ray $\left(L_{\mathrm{x}} \approx\right.$ $\left.10^{39} \mathrm{erg} \mathrm{s}^{-1}\right)$ and optical $\left(L_{\mathrm{opt}} \approx 3 \times 10^{38} \mathrm{erg} \mathrm{s}^{-1}\right)$ bands. The bright optical outbursts were qualitatively explained as X-ray reprocessing in a gas cloud surrounding the binary system.

Aims. Since then, further important information about the properties of A0538-66 have been obtained, and sophisticated photoionization codes have been developed to calculate the radiation emerging from a gas nebula illuminated by a central X-ray source. In the light of the new information and tools available, we considered it was worth studying again the enhanced optical emission displayed by A0538-66 to understand the mechanisms responsible for these unique events among the class of Be/X-ray binaries.

Methods. We performed about $10^{5}$ simulations of a gas envelope surrounding the binary system photoionized by an X-ray source. We assumed for the shape of the gas cloud either a sphere or a circumstellar disc observed edge-on. We studied the effects of varying the main properties of the envelope (shape, density, slope of the power law density profile, size) and the influence of different input X-ray spectra and X-ray luminosity on the optical/UV emission emerging from the photoionized cloud. We determined the properties of the cloud and the input X-ray emission by comparing the computed spectra with the $I U E$ spectrum and photometric $U B V$ measurements obtained during the outburst of 29 April 1981. We also explored the role played by the X-ray heating of the surface of the donor star and the accretion disc irradiated by the X-ray emission of the neutron star.

Results. We found that reprocessing in a spherical cloud with a shallow radial density distribution and size of about $3 \times 10^{12} \mathrm{~cm}$ can reproduce the optical/UV emission observed on 29 April 1981. To our knowledge, this configuration has never been observed either in A0538-66 during other epochs or in other Be/X-ray binaries. We found, contrary to the case of most other Be/X-ray binaries, that the optical/UV radiation produced by the X-ray heating of the surface of the donor star irradiated by the neutron star is non-negligible, due to the particular orbital parameters of this system that bring the neutron star very close to its companion.
\end{abstract}

Key words. accretion, accretion disks - stars: neutron - X-rays: binaries

\section{Introduction}

$\mathrm{Be} / \mathrm{X}$-ray binaries (Be/XRBs), first recognized as an independent class of X-ray sources by Maraschi et al. (1976), are the most conspicuous members of high-mass X-ray binaries (HMXBs; for a review, see e.g. Reig 2011). They consist of a Be star and, usually, a neutron star (NS). Most of them show a weak persistent X-ray emission sporadically interrupted by outbursts lasting from a few to several weeks. The X-ray outbursts are caused by the accretion onto the NS of the matter captured from the circumstellar disc around the Be star. Optical properties of $\mathrm{Be} / \mathrm{XRBs}$ have been studied since their discovery (e.g. Tarenghi et al. 1981), recently by using the extensive photometric observations of the Magellanic Clouds obtained by the MACHO and OGLE projects (e.g. Rajoelimanana et al. 2011; Bird et al. 2012; Schmidtke et al. 2013). The optical emission of $\mathrm{Be} / \mathrm{XRBs}$ is dominated by the radiation emitted at the surface of the Be star and by the circumstellar disc. Some Be/XRBs show long-term ( $200-\gtrsim 3000 \mathrm{~d}$ ) irregular or quasi-periodic variations, with amplitudes $\Delta m_{\mathrm{I}} \lesssim 0.6$ (Rajoelimanana et al. 2011).
This variability is likely linked to the formation and depletion of the circumstellar disc. The pattern of variability changes from source to source, and also depends on the inclination angle of the circumstellar disc with respect to the line of sight (see e.g. Haubois et al. 2012). A small group of Be/XRBs also shows orbital periodicities in the optical band with lower amplitudes $\left(\Delta m_{\mathrm{I}} \approx 0.01-0.2\right)$. This type of variability is usually attributed to the circumstellar disc perturbed by the orbiting NS. Unfortunately, a detailed model to explain the optical variability on orbital timescales is still missing.

A remarkable exception to the typical low-amplitude optical variability observed in Be/XRBs is 1A 0538-66 (hereafter A0538-66), which in 1981 showed recurrent optical outbursts with an increase of the optical brightness of $\approx 2.3$ magnitudes (Charles et al. 1983). A0538-66, located in the Large Magellanic Cloud (LMC), hosts a NS with a pulse period of $69 \mathrm{~ms}$ and orbital period of $\sim 16.6 \mathrm{~d}$ (Skinner et al. 1982), which corresponds to the recurrence period of the optical outbursts. It was discovered in 1977 (White \& Carpenter 1978) during an X-ray outburst that reached a peak luminosity of 


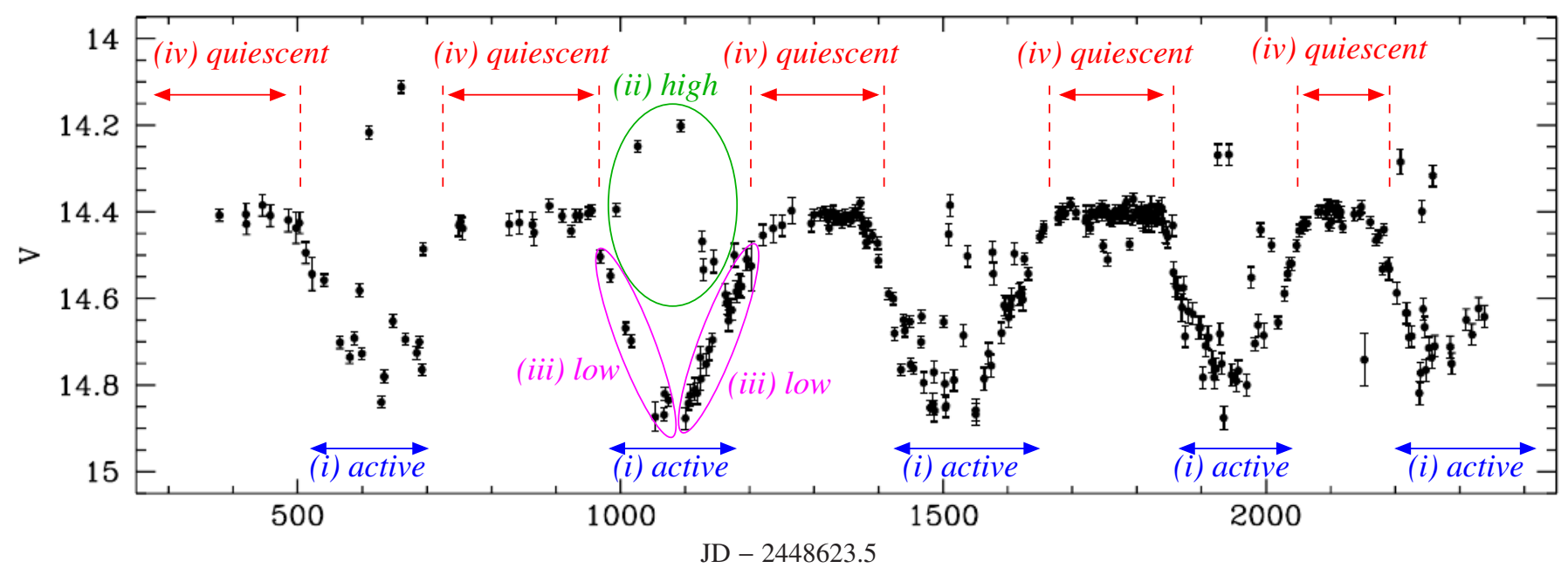

Fig. 1. $V$-band light curve of A0538-66 from MACHO project observations (figure adapted from Fig. 1 in Alcock et al. 2001). The quiescent, active states (low and high) of the McGowan \& Charles (2003) and Alcock et al. (2001) model are shown.

$8.5 \times 10^{38} \mathrm{erg} \mathrm{s}^{-1}$. Other super-Eddington outbursts were subsequently observed. ROSAT, BeppoSAX, ASCA, and XMMNewton (Campana et al. 2002; Kretschmar et al. 2004) revealed outbursts with lower luminosities $\left(L_{\mathrm{x}} \approx 10^{33}-10^{37} \mathrm{erg} \mathrm{s}^{-1}\right)$. The outbursts of A0538-66 show a wide range of durations, from a few hours to $\sim 14$ days, and a high dynamical range spanning five orders of magnitude. As mentioned above, A0538-66 displays a unique optical behaviour when compared with other Be/X-ray transients. Skinner $(1980,1981)$ discovered bright and recurrent optical outbursts (up to $L_{\mathrm{opt}} \approx 3 \times 10^{38} \mathrm{erg} \mathrm{s}^{-1}$; Charles et al. 1983 ) with period of $\sim 16.6 \mathrm{~d}$ in phase with the X-ray outbursts. A photometric light curve from 1915 to 1998, based on MACHO data and UK Schmidt and Harvard photographic $B$-band plates revealed, in addition to the recurrent outbursts with period $\sim 16.6 \mathrm{~d}$, a long-term modulation at $P_{\text {sup }}=420.8 \pm 0.8$ days, with a reddening at low fluxes, in agreement with the formation of a circumstellar disc (Alcock et al. 2001; McGowan \& Charles 2003). Alcock et al. (2001) and McGowan \& Charles (2003) suggested that the long-term modulation is caused by the depletion and formation of a circumstellar disc around the Be star, observed nearly edge-on. The orbital modulation is seen only at certain phases of the 421-day cycle, suggesting that $P_{\text {sup }}$ is caused by variations in the Be star envelope. When the NS is embedded in the high-density wind of the circumstellar disc (see active state in Fig. 1), it accretes material which leads to X-ray and optical outbursts (see high state in Fig. 1). This scenario is consistent with the observation of Balmer emission lines during the active state, testifying the presence of a circumstellar disc. When the optical flux is high for a long period (the quiescent state, see Fig. 1), the optical/UV spectrum does not show any Balmer emission, suggesting the absence of the circumstellar disc. Therefore, during the quiescent state, only the naked B star is observed. The exceptionally bright optical outbursts of A0538-66 observed until about 1981, were relatively long ( $t \approx 3$ days, see Fig. 1 in Densham et al. 1983). Subsequently, the source showed a different type of outbursts, faster $(t \lesssim 1 \mathrm{~d})$ and fainter $(\Delta m \approx 0.5)$, associated with X-ray outbursts with lower luminosity $\left(L_{\mathrm{x}}<10^{37} \mathrm{erg} \mathrm{s}^{-1}\right.$; Corbet et al. 1997; Alcock et al. 2001). Recently, it has been shown that A0538-66 still shows fast optical outbursts (Ducci et al. 2016; Rajoelimanana et al. 2017).

Densham et al. (1983), Maraschi et al. (1983), and Howarth et al. (1984), proposed a qualitative scenario to explain the bright optical outbursts observed in 1980 and 1981. The scenario requires that a dense gas cloud forms around the binary system from the material tidally displaced by the NS from the outer layers of the donor star over many orbits. The bright optical outbursts are then powered by the X-ray photons produced by the accreting NS which are reprocessed in the envelope. Maraschi et al. (1983) considered the models of high-density clouds illuminated by a central X-ray source proposed by Hatchett et al. (1976) and Kallman \& McCray (1982). Scaling the model calculations presented by these authors, Maraschi et al. (1983) found that the effective temperature of the optical radiation emitted during the optical outburst $\left(T \approx 10^{4} \mathrm{~K}\right.$ ) could be obtained assuming an X-ray luminosity of $L_{\mathrm{x}}=10^{39} \mathrm{erg} \mathrm{s}^{-1}$ and a homogeneous sphere of gas with density $n=10^{12} \mathrm{~cm}^{-3}$ and size of $R_{\text {out }}=3 \times 10^{12} \mathrm{~cm}$. Apparao \& Tarafdar (1988) used a similar approach to show that the enhanced continuum and emission lines of the optical outbursts are produced by a disc of gas around the Be star ionized by the X-ray radiation emitted by the NS during the periastron passage, when it crosses the circumstellar disc. Apparao \& Tarafdar (1988) used a model developed by Kallman \& McCray (1982), which consists of an X-ray source with a $10 \mathrm{keV}$ bremsstrahlung spectrum that ionizes a sphere with uniform density surrounding it. On the basis of this model, Apparao \& Tarafdar (1988) considered the ionized states of $\mathrm{H}$, $\mathrm{He}$, and $\mathrm{C}$ IV to calculate the continuum emission produced by an irradiated disc with height $5 \times 10^{11} \mathrm{~cm}$, a uniform density, and a given size. They calculated the continuum emission for different values of the input X-ray luminosity, density, and disc radius, assuming that the distance of the NS from the Be star at periastron is $5 \times 10^{12} \mathrm{~cm}$. Apparao \& Tarafdar (1988) found the best agreement between the computed emission and the observed ultraviolet and optical continuum of A0538-66 during an outburst for $L_{\mathrm{x}}=2.5 \times 10^{39} \mathrm{erg} \mathrm{s}^{-1}, n=2 \times 10^{11} \mathrm{~cm}^{-3}$, and $R_{\text {out }}=1.38 \times 10^{13} \mathrm{~cm}$.

The calculations presented by Maraschi et al. (1983) and Apparao \& Tarafdar (1988) were based on a simplified general class of models computed by Kallman \& McCray (1982) and Hatchett et al. (1976) proposed for a wide variety of astrophysical objects. Although they could be rescaled, they were not tailored for the specific case of A0538-66. In particular, we refer to the input X-ray spectrum, the geometry of the system, and the density properties of the nebula and the abundances, which 
were all fixed in the Kallman \& McCray (1982) model to values not suitable for the case of A0538-66. Therefore, we used the photoionization code CLOUDY (v17.01) to compute the emission spectrum of a cloud ionized by the $\mathrm{X}$-ray radiation produced by the accreting pulsar of A0538-66. CLOUDY is designed to simulate the physical conditions and emission spectra of a photoionized gas. It can be used to describe a wide variety of astrophysical situations (Ferland et al. 2017). The code requires as input the spectrum and luminosity of the X-ray source, the geometry of the cloud (we assume different sizes, shapes, and density profiles) and gas composition (we assume the typical metallicity of the LMC). The ionization and temperature structure are found by CLOUDY assuming a local balance between heating and cooling and between ionization and recombination. The processes taken into account include photoionization, Auger effect, Compton heating, charge transfer, collisional de-excitation, radiative and dielectronic recombination, bremsstrahlung, collisional ionization, and collisional excitation of bound levels. The radiation field is determined by solving the radiative transfer equation for both the continuum and line components (for further details see the documentation of CLOUDY ${ }^{1}$, and e.g. Ferland et al. 2017).

\section{Calculations}

We assumed that A0538-66 consists of a B1 III star with mass $M_{\mathrm{d}}=9 M_{\odot}$ and radius $R_{\mathrm{d}}=10 R_{\odot}$ and a NS with mass $M_{\mathrm{NS}}=$ $1.44 M_{\odot}$. The $M_{\mathrm{d}}=9 M_{\odot}$ value corresponds to the upper limit for the mass of the donor star derived by Rajoelimanana et al. (2017) from the mass function assuming $M_{\mathrm{NS}}=1.44 M_{\odot}$ and from the absence of an X-ray eclipse. Because of the lack of information needed to determine the mass function of the X-ray pulsar, the two masses cannot be measured separately. Therefore, the values we assumed for $M_{\mathrm{d}}$ and $M_{\mathrm{NS}}$ may slightly deviate from the real values of the system. For the orbital parameters we assumed $P_{\text {orb }}=16.6409 \mathrm{~d}$ and eccentricity $e=0.72$ (Rajoelimanana et al. 2017).

Simulations are divided into three main groups. In each group, we considered a different input spectrum:

- bremsstrahlung, with temperature $k T=10 \mathrm{keV}$;

- black body, with temperature $k T=2.4 \mathrm{keV}$;

- Comptonization of soft photons in a hot plasma (сомpтT in Xspec; Titarchuk 1994; Arnaud 1996), with input soft photon (Wien law) temperature $k T_{0}=1.35 \mathrm{keV}$, plasma temperature $k T=5.5 \mathrm{keV}$, and plasma optical depth $\tau=17$ (based on the spectrum of V0332+53 during an outburst of $L_{\mathrm{x}} \approx 10^{38} \mathrm{erg} \mathrm{s}^{-1}$; see Doroshenko et al. 2017).

For the first group, we considered a bremsstrahlung model with temperature $k T=10 \mathrm{keV}$ to allow us to compare our results with those obtained by Apparao \& Tarafdar (1988), although the results from this model should be taken with caution because of the large fraction of soft X-ray and UV photons it produces compared to more physical models (see Fig. 2). For the second group of simulations, we considered the model found by Ponman et al. (1984) that best fits the X-ray emission from A0538-66 during a bright outburst observed by the Einstein satellite. For the third group, we considered a physical model with properties similar to those observed in other Be/XRBs observed during highluminosity states. For each of these groups, we considered two geometries for the envelope: a sphere or a disc with uniform height $h=5 \times 10^{11} \mathrm{~cm}$ (similarly to Apparao \& Tarafdar 1988).

We considered for our calculations the closed geometry used in CLOUDY because we expect that the covering factor of the

\footnotetext{
1 http://wWw. nublado.org/
}

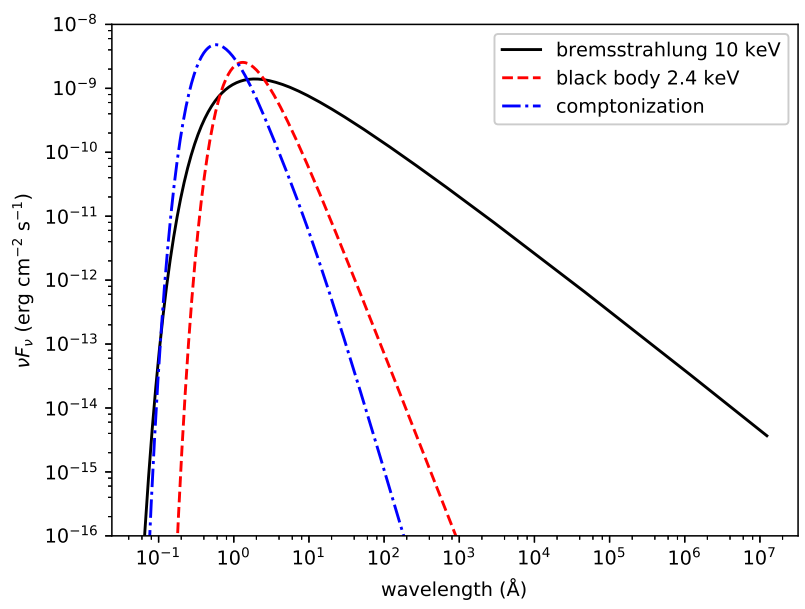

Fig. 2. Comparison between the input spectra used to compute the spectrum emerging from the gas cloud illuminated by the NS and the donor star. The luminosity in the energy range $1-20 \mathrm{keV}$ is the same for all the input spectra $\left(L_{\mathrm{x}}=10^{39} \mathrm{erg} \mathrm{s}^{-1}\right)$.

cloud seen by the X-ray source is large for the spherical and for the disc case. On the basis of previous findings, we assumed that the circumstellar disc is observed edge-on. We adopted for all the simulations a metallicity of $Z=0.4 Z_{\odot}$, according to the average values of LMC reported in Zhukovska \& Henning (2013) and Russell \& Dopita (1992). The ionization parameter inside the cloud (according to the definition of Tarter et al. 1969) in our simulations is $\lesssim 10^{6} \mathrm{erg} \mathrm{cm} \mathrm{s}^{-1}$.

Optical and near-infrared studies showed that the disc density of all Be stars (isolated or in Be/XRBs) follows a power-law density distribution,

$n(r)=n_{0}\left(\frac{r}{R_{\mathrm{d}}}\right)^{-\alpha} \mathrm{cm}^{-3}$,

where $n_{0}$ is the number density of atoms at $R_{\mathrm{d}}$ and $\alpha$ is the density slope (Waters et al. 1989). We used Eq. (1) to describe the density distribution also in the case of clouds with spherical symmetry.

For each group and geometry (bremmstrahlung, black body, Comptonization; sphere or disc) we ran $10^{3}$ simulations varying $n_{0}$, the size of the cloud $\left(R_{\text {out }}\right)$, and $\alpha$ in the ranges $10^{11} \leq$ $n_{0} \leq 10^{13} \mathrm{~cm}^{-3}, 2 \times 10^{12} \leq R_{\text {out }} \leq 3 \times 10^{13} \mathrm{~cm}, 0 \leq \alpha \leq 2.5$ in a grid $10 \times 10 \times 10$. The assumed values of $R_{\text {out }}$ and $n_{0}$ were uniformly distributed in a $\log _{10}$ space in the ranges defined above, while $\alpha$ values were uniformly distributed in a linear space. Although the X-ray luminosity $L_{\mathrm{x}}$ at the peak of the outbursts is reasonably well constrained from the observations (Ponman et al. 1984), A0538-66 was not observed in X-rays during the outburst of 29 April 1981. Therefore, similarly to Apparao \& Tarafdar (1988), we also left this parameter free to vary in the range $10^{38} \leq L_{\mathrm{x}} \leq 5 \times 10^{39} \mathrm{erg} \mathrm{s}^{-1}$.

As mentioned above, we compared each spectrum computed with CLOUDY with the $I U E$ spectrum and $U B V$ magnitudes of the outburst of 29 April 1981 reported in Charles et al. (1983). The intensities of all spectral lines provided by CLOUDY are calculated correctly, although the default code does not compute spectral line profiles ${ }^{2}$. In addition, CLOUDY does not take into account the wind velocity; therefore, effects like broadening of the line width or wavelength shift caused by the outflowing motion of the gas in the wind are not reproduced in

\footnotetext{
2 https://wwW . nublado.org/wiki/FaqPage
} 
Table 1. Best fit results and the relative reduced chi-square values $\left(\chi_{v}^{2}\right)$ for the comparison between the optical/UV spectra computed with CLOUDY and the IUE+UBV spectrum of the outburst of 29 April 1981.

\begin{tabular}{|c|c|c|c|c|c|c|}
\hline Input spectrum & $\begin{array}{c}R_{\text {out }} \\
\log _{10}(\mathrm{~cm})\end{array}$ & $\begin{array}{c}n_{0} \\
\log _{10}(\mathrm{~cm}) \\
\end{array}$ & $\alpha$ & $\begin{array}{c}\chi_{v}^{2} \\
\text { (13 d.o.f.) }\end{array}$ & $\begin{array}{c}\mathrm{C}_{\mathrm{IV}} \lambda 1550 \\
\log _{10}\left(\mathrm{erg} \mathrm{s}^{-1}\right)\end{array}$ & $\begin{array}{c}\text { He II } \lambda 1640 \\
\log _{10}\left(\mathrm{erg} \mathrm{s}^{-1}\right)\end{array}$ \\
\hline Bremmstrahlung & $12.42 \pm 0.05$ & $12.78 \pm 0.11$ & $0.60 \pm 0.14$ & 2.12 & 35.8 & 36.1 \\
\hline Black body & $12.42 \pm 0.05$ & $12.78 \pm 0.11$ & $0.60 \pm 0.14$ & 1.32 & 36.2 & 35.9 \\
\hline Comptonization & $12.63 \pm 0.07$ & $12.78 \pm 0.11$ & $1.11 \pm 0.14$ & 1.44 & 36.2 & 36.2 \\
\hline
\end{tabular}

Notes. The best fit parameters were obtained assuming $L_{\mathrm{x}}=2.5 \times 10^{38} \mathrm{erg} \mathrm{s}^{-1}(1-20 \mathrm{keV})$ and a spherical geometry.

the computed spectrum. We thus decided to compare the computed and observed continuum in the region of the spectrum above $2000 \AA$, which has few prominent emission lines. Then we compared the luminosities of the emission lines $C$ iv $\lambda 1550$ and $\mathrm{He}$ II $\lambda 1640$ computed by CLOUDY with the values reported in Apparao \& Tarafdar (1988): $L_{\mathrm{C}_{\text {IV }} \lambda 1550}=3 \times 10^{36} \mathrm{erg} \mathrm{s}^{-1}$; $L_{\mathrm{He} \text { II } \lambda 1640}=8 \times 10^{36} \mathrm{erg} \mathrm{s}^{-1}$. For the comparison between the computed and observed continuum, we used the $\chi^{2}$ test. The $\chi^{2}$ value is computed at each grid point for all the parameters $(\alpha$, $\left.n_{0}, R_{\mathrm{d}}, L_{\mathrm{x}}\right)$. Then the global minimum over the grid is found at the grid point with the lowest value. The corresponding $\alpha, n_{0}$, $R_{\mathrm{d}}$, and $L_{\mathrm{x}}$, are assumed to be the best solutions. We do not use interpolation between grid points in the process.

\section{Results and discussion}

\subsection{Optical/UV emission from a gas cloud ionized by the $X$-rays from the NS}

We first consider the bright outbursts of A0538-66 under the assumption that they are caused by reprocessing of X-rays radiation in the envelope surrounding the binary system. When we assumed a circumstellar disc for the gas cloud, we found that the luminosities of the lines $\mathrm{C}_{\text {IV }} \lambda 1550$ and $\mathrm{He}$ II $\lambda 1640$ were 100-1000 times fainter than those observed, and therefore this geometry was discarded. Instead, for a spherical shell we found more acceptable values. The difference between the luminosities of these lines in the spherical and disc cases probably arises because a sphere absorbs more energy from the source than a disc, and thus can reradiate it more in the form of lines. Therefore, we list in Table 1 only the best fit solutions obtained for a spherical gas shell. Errors in Table 1 are at the $1 \sigma$ confidence level and are calculated for each parameter independently according to the method described, for example, in Avni (1976). Uncertainties in Table 1 are comparable with the grid cell size, and as such should be taken with caution. Although the reduced $\chi^{2}$ value $\left(\chi_{v}^{2}\right)$ for the bremsstrahlung case is greater than two, the null hypothesis probability is 0.0104 . Hence, for this case, the data and model are consistent, though only at the $\approx 99 \%$ confidence level. For the black body case there are two additional solutions with $\chi_{v}^{2}<2$ (but worse than the best fit solution shown in Table 1) for two sets of parameters: $\alpha=1.11 \pm 0.14$, $n_{0}=12.78 \pm 0.11, R_{\text {out }}=12.63 \pm 0.07\left(\chi_{v}^{2}=1.33,13\right.$ d.o.f. $)$; $\alpha=1.67 \pm 0.14, n_{0}=13.00 \pm 0.11, R_{\text {out }}=13.34 \pm 0.08$ $\left(\chi_{v}^{2}=1.44,13\right.$ d.o.f.; errors at $1 \sigma$ confidence level $)$. In both cases, $L_{\mathrm{x}}=2.5 \times 10^{38} \mathrm{erg} \mathrm{s}^{-1}(1-20 \mathrm{keV})$.

Figure 3 shows the $\chi_{v}^{2}$ values as a function of the fit parameters $R_{\text {out }}, n_{0}$, and $\alpha$ for each input spectrum (Column 1: bremsstrahlung; Column 2: black body; Column 3: Comptonization). Each panel shows the lowest values of the $\chi_{v}^{2}$ as a function of two of the three parameters mentioned above. In all cases, $L_{\mathrm{x}}$ is fixed to the best fit value $2.5 \times 10^{38} \mathrm{erg} \mathrm{s}^{-1}$. Figure 4 shows, for each type of input spectrum, the computed spectra which best fit the observed spectrum (see Table 1). These best fit spectra seem to underestimate the observed flux at $\lambda \gtrsim 4000 \AA$. The mismatch for the $V$ magnitude corresponds to about $2.5 \sigma$. This discrepancy might indicate that the models we assumed for our calculations are too simple. Further considerations that can be taken into account in future works are the degree of inhomogeneity of the cloud and the presence of other radiation fields in the system, such as the intrinsic radiation emission by the donor star, possibly modified by the irradiation flux from the pulsar impinging the donor star surface (see Sect. 3.2), and the circumstellar disc itself, which emits radiation caused by viscosity (Lee et al. 1991).

The gas cloud around the system and the X-ray reprocessing caused by it also affect the emerging (observable) spectrum in the X-ray band. As an example, we show in Fig. 5 a comparison between the $k T=2.4 \mathrm{keV}$ black-body spectrum (Fig. 5, top panel, dashed blue line) used as input in the CLOUDY simulations, and the X-ray spectrum emerging from the ionized nebula (top panel, solid red line) for the best fit parameters in Table 1. To check whether the distortion of the emerging spectrum could have been detected by the previous observations performed by the Monitor Proportional Counter (MPC) on board the Einstein satellite, we simulated the emerging X-ray spectrum of A0538-66 computed with CLOUDY (solid red line in the top panel of the same figure) using the available Einstein/MPC detection redistribution matrices ${ }^{3}$, with an exposure time of $4328 \mathrm{~s}$ (see Ponman et al. 1984). We obtained a good fit $\left(\chi_{v}^{2}=\right.$ $1.04,5$ d.o.f.) assuming an absorbed $\left(N_{\mathrm{H}}=9.7_{-3.5}^{+8.1} \times 10^{23} \mathrm{~cm}^{-2}\right)$ black body $(k T=2.2 \pm 0.6 \mathrm{keV}$; errors at $1 \sigma$ confidence level). The result is shown in Fig. 5. The output temperature of the black body is only marginally different from the input temperature, while the column density is about a factor of five higher than that measured by Ponman et al. (1984), but we note that the latter is subject to a large uncertainty due to the limited energy range of the Einstein/MPC.

In the years following the bright optical outbursts studied in this work, A0538-66 showed fainter and shorter optical outbursts, while the $\mathrm{H} \alpha$ line profiles and the long-term photometric variability indicated the presence of a circumstellar disc. To check whether the properties of these weaker optical outbursts can be explained by a photoionized circumstellar disc illuminated by an X-ray source, we assumed the same orbital and stellar parameters described in Sect. 2. For the gas cloud, we assumed the typical values reported in Rivinius et al. (2013) for the circumstellar discs in Be/XRBs, namely $n_{0}=6 \times 10^{12} \mathrm{~cm}^{-3}$ $\left(\rho_{0}=n_{0} m_{\mathrm{p}}=10^{-11} \mathrm{~g} \mathrm{~cm}^{-3}\right)$ and $R_{\text {out }}=8 R_{\mathrm{d}} \approx 5.6 \times 10^{12} \mathrm{~cm}$. Typically, $\alpha$ in Be stars is in the range $3-4$. However, in Be/XRBs the circumstellar disc can be truncated by tidal interactions with

\footnotetext{
3 ftp://legacy.gsfc.nasa.gov/caldb/data/einstein/mpc/ $\mathrm{cpf}$
} 

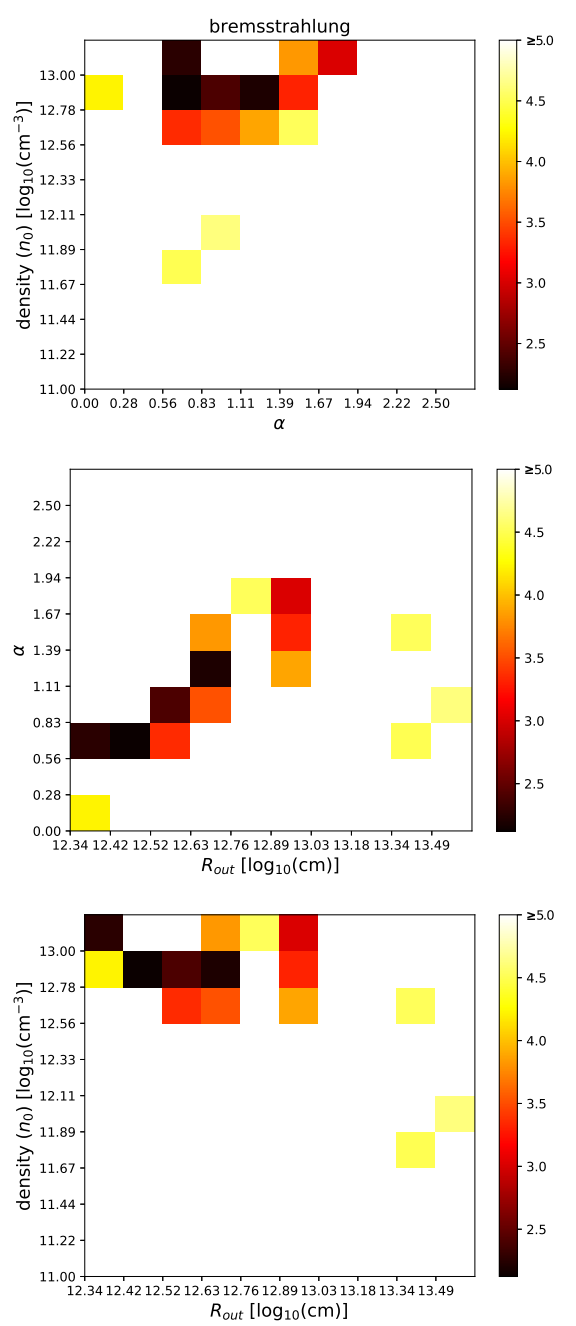
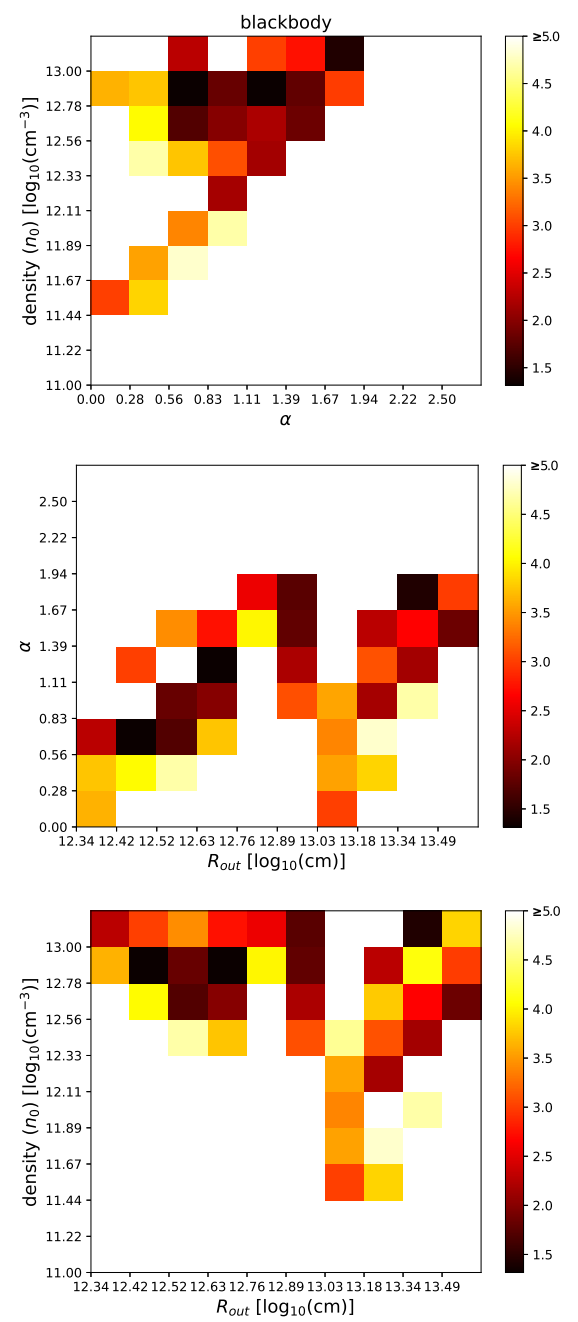
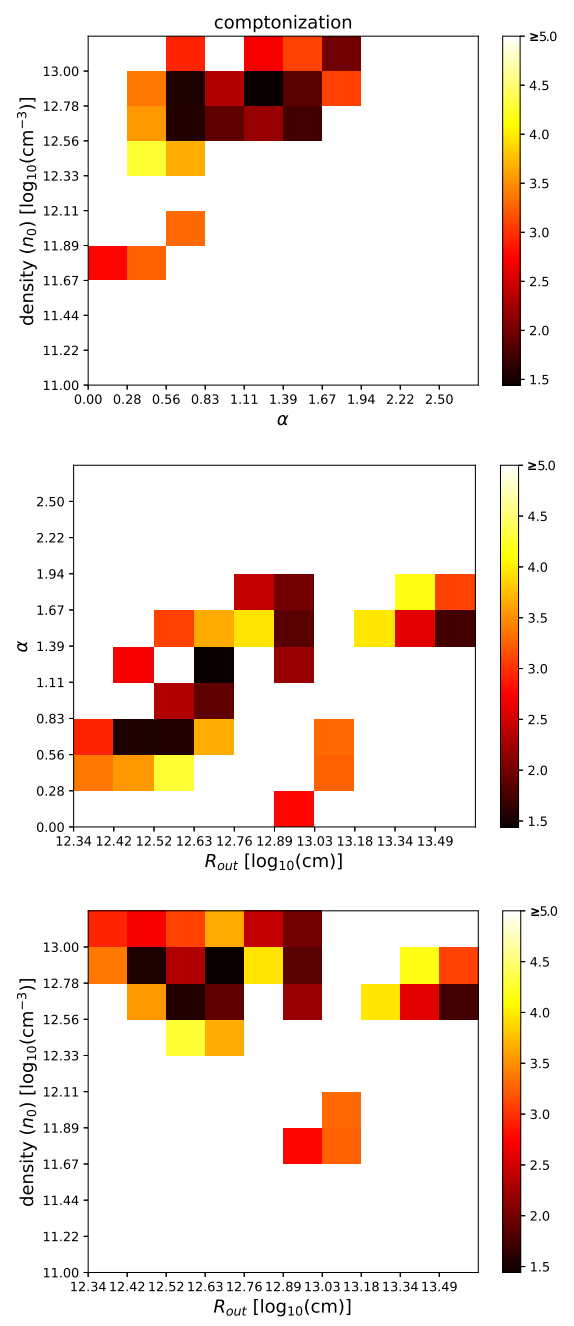

Fig. 3. $\chi_{v}^{2}$ values as a function of the parameters $R_{\mathrm{out}}, n_{0}$, and $\alpha$ for three different input spectra. In all cases $L_{\mathrm{x}}=2.5 \times 10^{38} \mathrm{erg}, \mathrm{s}^{-1}(1-20 \mathrm{keV})$.
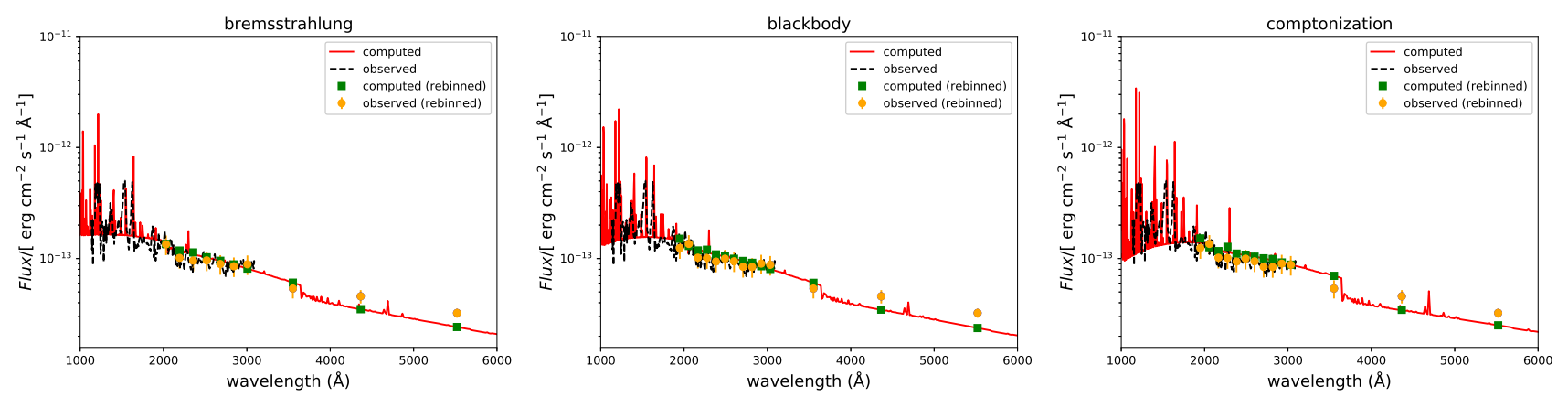

Fig. 4. IUE spectrum and $U B V$ photometric fluxes taken during the observation of the outburst of A0538-66 of 29 April 1981 , and the best fit spectra computed with CLOUDY assuming the parameters shown in Table 1 for three different input spectra.

the NS. This leads to a shallower density profile. Therefore, we assumed the conservative value of $\alpha=3$ found in other $\mathrm{Be} / \mathrm{XRBs}$ (Rivinius et al. 2013). For the input spectrum we used a black body with temperature $k T=2.4 \mathrm{keV}$ and $1-20 \mathrm{keV}$ luminosity ranging from $5 \times 10^{37} \mathrm{erg} \mathrm{s}^{-1}$ to $10^{40} \mathrm{erg} \mathrm{s}^{-1}$. We considered the case of a disc seen edge-on in agreement with the results from Alcock et al. (2001) and McGowan \& Charles (2003). The results are shown in Fig. 6 where the difference in magnitude $\Delta V$ between the active low state $(V=15$, i.e. we assumed the lowest magnitude from Fig. 1) and outburst is plotted for each input X-ray luminosity. Also shown in the figure are two unusual scenarios for Be/XRBs, having density profiles with extreme slopes, $\alpha=2.5$ and $\alpha=2$. For comparison, we plotted $\Delta V$ observed on 29 April 1981 (Charles et al. 1983). Figure 6 shows that for a density profile of $\alpha=3$, the increase in the $V$ magnitude during the outburst is negligible compared to the magnitude of the system star plus circumstellar disc observed during the active-low state. This reinforces our previous findings that the gas cloud around the system on 29 April 1981 was not a classical circumstellar disc and, albeit indirectly, supports the lack of bright optical outbursts associated with the X-ray outbursts in the other Be/XRBs. For $\alpha=2$ and $\alpha=2.5$ there is 

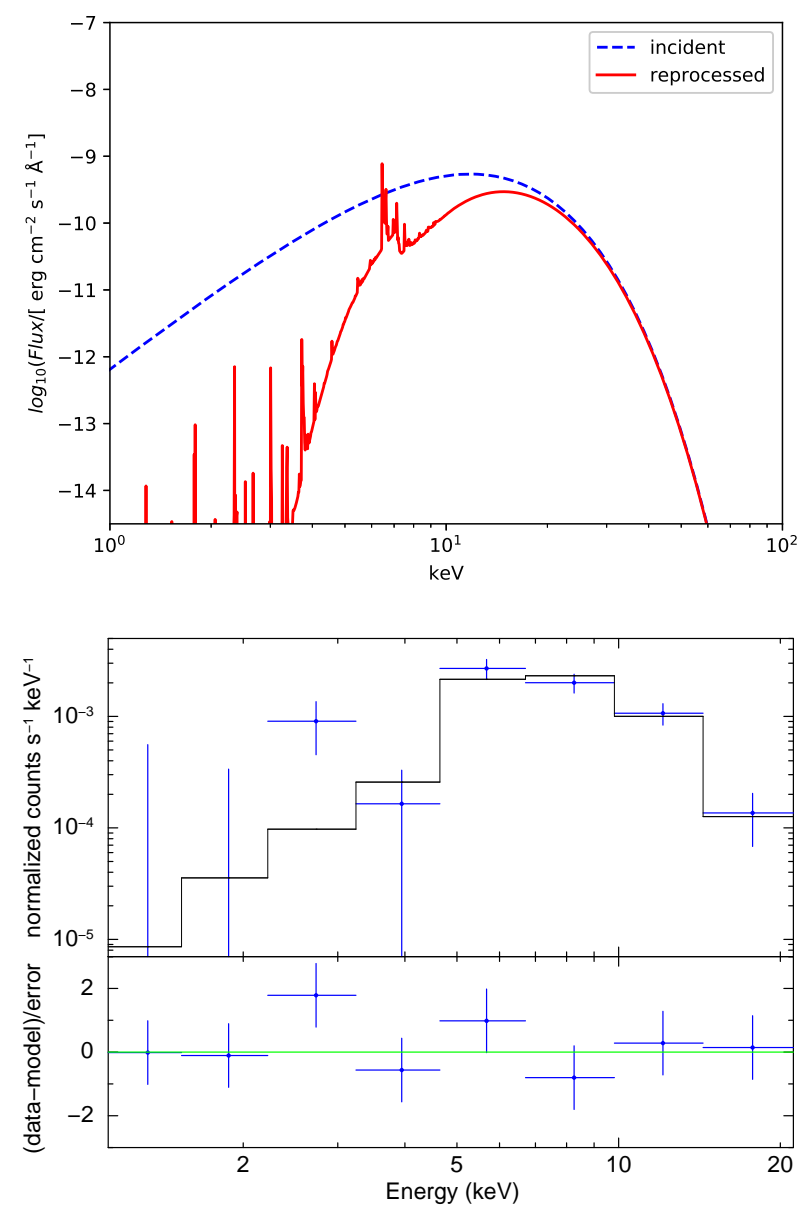

Fig. 5. Top panel: comparison between the $k T=2.4 \mathrm{keV}$ black-body spectrum adopted as input in our CLOUDY simulations (blue dashed line) and the spectrum emerging from the photoionized circumstellar disc seen edge-on (red solid line). Bottom panel: simulated spectrum of A0538-66 based on the spectrum emerging from the nebula, after reprocessing took place (solid red line from top panel), for a $4328 \mathrm{~s}$ Einstein/MPC observation fitted with an absorbed $\left(N_{\mathrm{H}}=9.7_{-3.5}^{+8.1} \times\right.$ $\left.10^{23} \mathrm{~cm}^{-2}\right)$ black body $(k T=2.2 \pm 0.6 \mathrm{keV}$; errors at $1 \sigma$ confidence level).

an increase of $\Delta V \approx 0.5-1.5$, similar to that seen in the lowluminosity optical outbursts displayed by A0538-66 (see Fig. 1 and Ducci et al. 2016). Although these steep density profiles are not observed in $\mathrm{Be} / \mathrm{XRBs}$, it is important to note that a value of $\alpha=2.1$ was measured by Waters et al. (1988) in A0538-66 during an infrared observation on 9 October 1981 carried out during an outburst not observed in the optical bands. Nonetheless, Waters et al. (1988) were able to extrapolate an expected $V$ magnitude during the outburst of $V \approx 13.6$, which is in good agreement with the value at the peak of the red line $(\alpha=2)$ in Fig. 6.

\subsection{X-ray irradiation of the surface of the donor star}

In the previous section, we considered the case of optical outbursts powered by the reprocessing of X-ray radiation in the envelope surrounding the binary system. However, as already noticed by Charles et al. (1983), other mechanisms could cause the optical outbursts. Here we consider the effects of the irradiation of the surface of the Be star by X-ray photons emitted by the accreting NS. Because of the high eccentricity and small

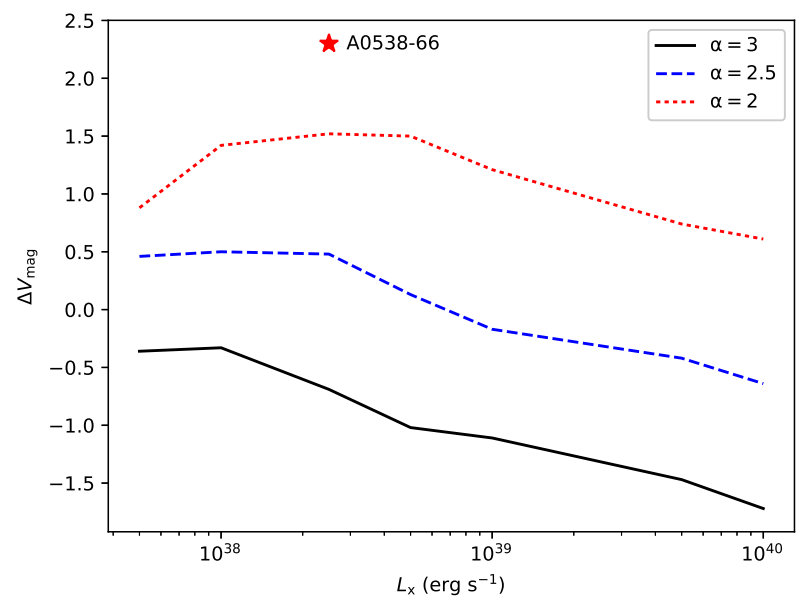

Fig. 6. $\Delta V_{\operatorname{mag}}$ as a function of the input $\mathrm{X}$-ray luminosity $(1-20 \mathrm{keV})$ for a black-body input spectrum with $k T=2.4 \mathrm{keV}$ and three different slopes of the density profile of the circumstellar disc. The red star shows the $\Delta V_{\text {mag }}$ of the 29 April 1981 outburst.

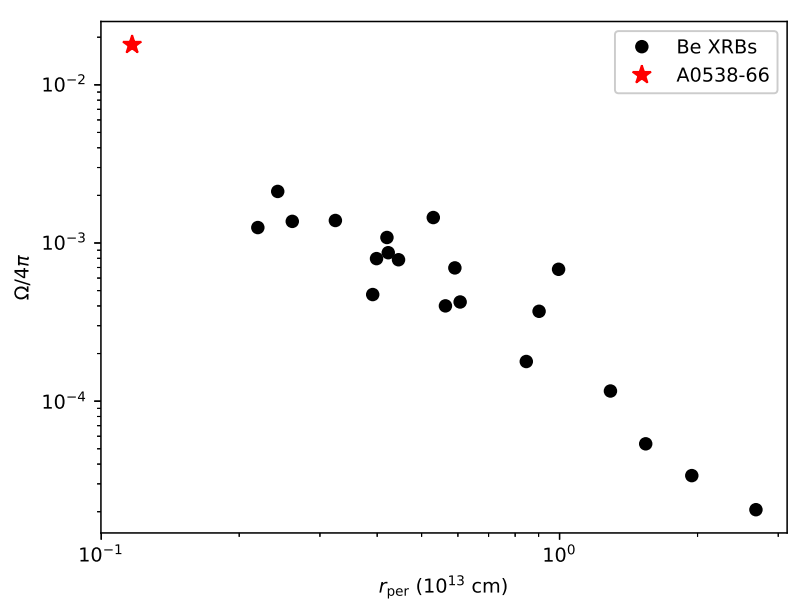

Fig. 7. Fraction of solid angle of the X-ray radiation emitted by the NS and intercepted by the donor star at periastron, for different Be/XRBs with known orbital parameters, compared to A0538-66.

orbital period of A0538-66, the separation between the two stars at periastron is such that the fraction of X-ray radiation emitted by the NS (assuming isotropic emission) intercepted by the companion star is higher than in the other Be/XRBs with known orbital parameters. This characteristic can be seen in Fig. 7, where we show-for a sample of Be/XRBs with known system parameters (orbital period, eccentricity, mass, and radius of the donor star or its spectral type) - the fraction of NS radiation intercepted by the donor star at periastron as a function of the separation of the stars at periastron. We considered for this plot the binary systems with OBe stars reported in the catalogue of Liu et al. (2006) and Table 2 of Klus et al. (2014) with known orbital period and eccentricity, spectral type, and luminosity class of the donor star. In some cases the masses and radii of the donor stars were reported in Okazaki \& Negueruela (2001). In the other cases, we derived them from the spectral and luminosity class using the Catalogue of Apparent Diameters and Absolute Radii of Stars (CADARS; Pasinetti Fracassini et al. 2001) and the catalogue of Hohle et al. (2010).

We calculated the expected optical/UV spectrum emitted by the surface of the donor star irradiated by the NS at the periastron passage using a model developed to study these effects in binary 


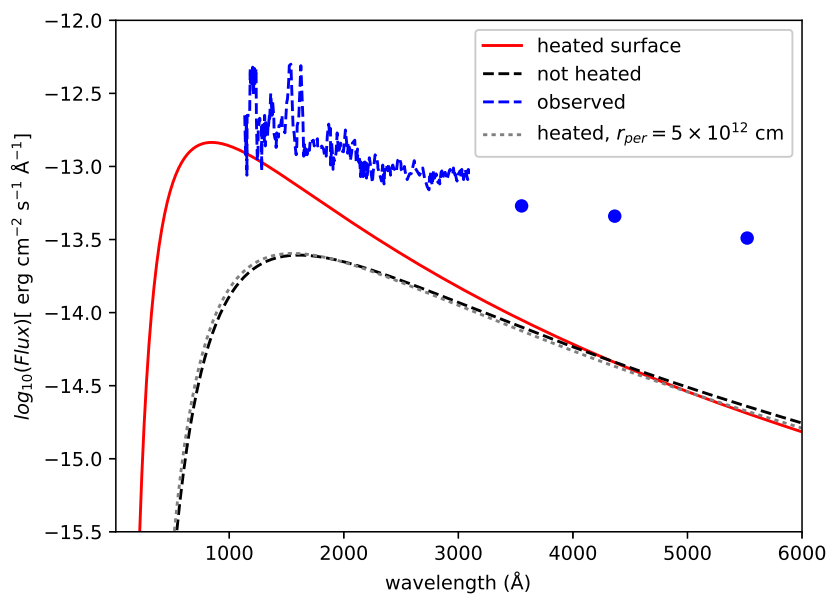

Fig. 8. Comparison between the optical/UV spectrum of the donor star of A0538-66 not irradiated by the NS and the heated spectrum for the irradiated surface of the donor star. We assumed here $\eta=0.5$ and an input X-ray luminosity of $L_{\mathrm{x}}=10^{39} \mathrm{erg} \mathrm{s}^{-1}$.

systems that hosts a bright accreting X-ray source (e.g. Beech 1985 and Tjemkes et al. 1986). In this model, the X-rays emitted by the compact object are absorbed by the atmosphere of the companion star and then reradiated at lower energy. For each surface element of the Be star, the local effective temperature increases according to

$T_{i}^{4}=T_{\mathrm{e}, i}^{4}+(1-\eta) \frac{L_{\mathrm{x}} \cos \phi}{4 \pi \sigma_{\mathrm{SB}} r^{2}}$,

where $T_{\mathrm{e}, \mathrm{i}}$ is the effective temperature of the surface element in the absence of $\mathrm{X}$-ray irradiation, $\eta$ is the fraction of reflected $\mathrm{X}$-rays (albedo), $L_{\mathrm{X}}$ is the luminosity of the $\mathrm{X}$-ray source (assumed to be emitted isotropically from the NS), $\sigma_{\mathrm{SB}}$ is the Stefan-Boltzmann constant, $\phi$ is the angle between the normal to the surface element and the direction to the X-ray source, and $r$ is the distance from the X-ray source to the surface element. In our calculations, we neglect gravity darkening effects and we assume a spherically symmetric donor star and $\eta=0.5$. The same value for the albedo was assumed by Charles et al. (1983) to calculate the effects of Xray heating on the Be star of A0538-66. In our calculations, the total spectrum and luminosity of the heated side of the companion is then determined by the sum of the black-body spectra emitted by each surface element. Figure 8 shows the spectrum irradiated (red line) by the heated side of the donor star during the periastron passage of the NS emitting at $L_{\mathrm{x}}=10^{39} \mathrm{erg} \mathrm{s}^{-1}$ compared to the undisturbed spectrum of the donor star $\left(T_{\mathrm{eff}}=18000 \mathrm{~K}\right.$, $L_{\text {opt }}=4.7 \times 10^{37} \mathrm{erg} \mathrm{s}^{-1}$, from Maraschi et al. 1983) and the $I U E+U B V$ spectrum. We also show in the same figure that the $\mathrm{X}$ ray heating would be negligible if the NS were located at a greater distance, for example, $r_{\mathrm{per}}=5 \times 10^{12} \mathrm{~cm}$, which is the average periastron separation of the Be/NS binaries (dotted grey line). In conclusion, the X-ray heating of the surface of the donor star of A0538-66 is non-negligible, but it is not enough to explain alone the IUE+UBV spectrum observed during the bright optical outburst of 29 April 1981.

\subsection{X-ray heating of the surface of an accretion disc}

Charles et al. (1983) ruled out the possibility that the UV and optical emission of A0538-66 during the outburst was produced by a Shakura-Sunyaev disc, for which the known relation $F_{v} \propto v^{1 / 3}$ is expected. However, the spectral shape of an

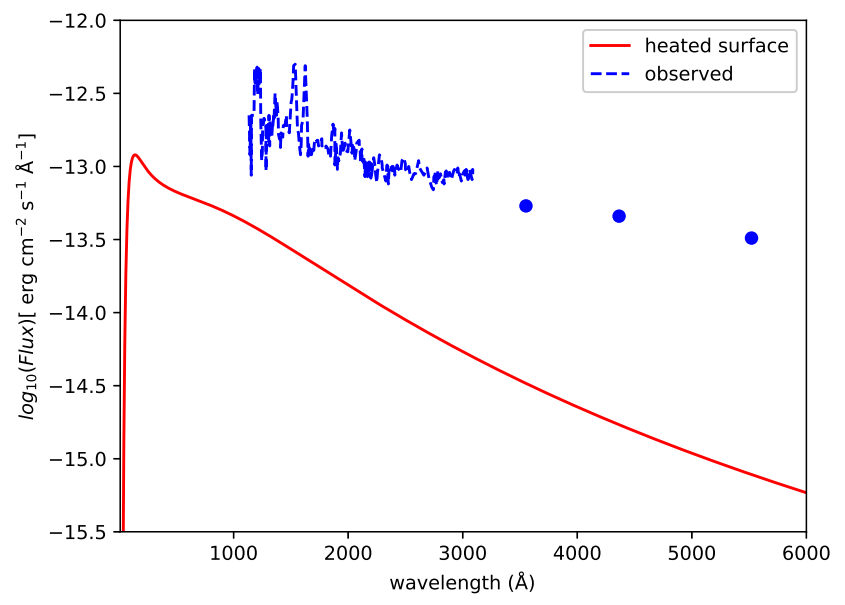

Fig. 9. Comparison between the observed optical/UV spectrum of A0538-66 during an outburst and the calculated spectrum from an irradiated accretion disc.

accretion disc irradiated by the X-ray photons from the accreting NS is significantly different from $F_{v} \propto v^{1 / 3}$ (see e.g. the review by Hynes 2010). Although it is not clear whether an accretion disc was actually present during the optical outburst studied in this work, it has recently been argued that an accretion disc might be present during the most recent periastron passages (Rajoelimanana et al. 2017). It is therefore worth examining this case. We followed the calculations proposed by Vrtilek et al. (1990) to model the spectrum of an irradiated disc. In their model, the temperature of the irradiated disc at a radius $r$ is given by

$T(r)=\left[\frac{f L_{\mathrm{x}} \sqrt{k G M_{\mathrm{NS}} /\left(\mu \mathrm{m}_{\mathrm{p}}\right)}}{14 \pi \sigma_{\mathrm{SB}} G M_{\mathrm{NS}}}\right]^{2 / 7}$,

where $f=0.5$ is the fraction of the $\mathrm{X}$-ray radiation absorbed by the disc surface, $k$ is the Boltzmann constant, $\mu=0.5$ is the mean molecular weight, $m_{\mathrm{p}}$ is the proton mass, $L_{\mathrm{x}}$ is the X-ray luminosity of the NS, $G$ is the gravitational constant, and $M_{\mathrm{NS}}$ is the mass of the NS. We assume that the spectrum of the ring of the accretion disc at radius $r$ is described by a black body with temperature given by Eq. (3). The spectrum of the entire accretion disc is then given by the sum of all the ring spectra. We calculated the emission from an irradiated disc assuming different values of the input X-ray luminosity, without finding an appropriate solution. As an example, Fig. 9 shows a comparison between the observed UV and optical emission of A0538-66 during the outburst and the calculated continuum emission of an accretion disc with size $R_{\text {disc }}=3 \times 10^{11} \mathrm{~cm}$ irradiated by a NS emitting at $L_{\mathrm{x}}=10^{39} \mathrm{erg} \mathrm{s}^{-1}$.

\section{Conclusions}

We examined different mechanisms to explain the bright optical outbursts shown by A0538-66. We found, through CLOUDY simulations, that the X-ray photoionization of a spherical shell around the binary system can explain reasonably well the observed $I U E$ spectrum and the $U B V$ magnitudes taken during the outburst of 29 April 1981. We found the best fit between the computed and observed spectra by assuming for the input X-ray spectrum a black body with temperature of $k T=2.4 \mathrm{keV}$ and a $1-20 \mathrm{keV}$ luminosity of $L_{\mathrm{x}}=2.5 \times 10^{38} \mathrm{erg} \mathrm{s}^{-1}$ (i.e. the spectrum observed by Ponman et al. (1984) during another outburst 
that reached similar X-ray luminosities). On the basis of our simulations, the properties of the gas cloud of A0538-66 during the bright optical outbursts seem consistent with the qualitative scenario proposed by Densham et al. (1983), Howarth et al. (1984), and Maraschi et al. (1983), where a spherical gas cloud forms around the binary system from the material tidally displaced by the NS from the outer layers of the donor star over many orbits. The bright optical outbursts are then powered by the X-ray photons produced by the accreting NS, which are reprocessed in the envelope. We found that our simulations can explain the density profile and $V$ magnitude measured by Waters et al. (1988) during the outburst of 9 October 1981. Nonetheless, there are some points in our results that need to be clarified:

- the column density value obtained from the fit of the X-ray spectrum (1-20 keV) emerging from the photoionized nebula with an absorbed black body (see Sect. 3.1 and Fig. 5) is about five times larger than the highest value measured by Ponman et al. (1984). However, Ponman et al. (1984) noted that the column density of A0538-66 was highly variable during the outburst. In addition, we note that the $\chi_{v}^{2}=1.633$ (3 d.o.f.) reported in Table 1 of Ponman et al. (1984) shows that the assumed fitting model (absorbed black body) does not describe well the Einstein/MPC data, and the uncertainties on the spectral parameters were not reported;

- the luminosities of the UV lines C IV $\lambda 1550$ and He II $\lambda 1640$ reported in Table 1 are, within an order of magnitude, in agreement with those observed; however, their luminosity is systematically lower than that observed;

- the best fit spectra shown in Fig. 4 seem to underestimate the observed flux at $\lambda \gtrsim 4000 \AA$.

These issues could indicate either that the photoionization of a cloud surrounding the system is not the correct explanation for the observed optical outbursts, or that our model is incomplete. In this regard, we have shown that another peculiarity of A0538-66 with respect to the other $\mathrm{Be} / \mathrm{XRBs}$ is that the radiation produced by the $X$-ray heating of the surface of the donor star is non-negligible during the bright X-ray outbursts (Sect. 3.2). Although this mechanism cannot explain alone the observed UV/optical emission during the bright outbursts, we cannot exclude, for example, that the reprocessing in the gas cloud around the system of the radiation emitted by the heated surface of the donor may account for the missing optical emission mentioned above. Future investigations based on more complex models will help to explain the cause of the small discrepancies with the observations that we have reported here.

Acknowledgements. We thank the anonymous referee for the useful comment that helped to improve the paper. We acknowledge support from the High Performance and Cloud Computing Group at the Zentrum für Datenverarbeitung of the University of Tübingen, the state of Baden-Württemberg through bwHPC and the German Research Foundation (DFG) through grant No. INST 37/9351 FUGG. SM and PR acknowledge financial contribution from the agreement ASI-INAF n.2017-14-H.0. PR acknowledges contract ASI-INAF I/004/11/0. KH is grateful to the Polish National Science Center for support under grant No. 2015/17/B/ST9/03422.

\section{References}

Alcock, C., Allsman, R. A., Alves, D. R., et al. 2001, MNRAS, 321, 678 Apparao, K. M. V., \& Tarafdar, S. P. 1988, ApJ, 335, 147

Arnaud, K. A. 1996, Astronomical Data Analysis Software and Systems V, eds. G. H. Jacoby, \& J. Barnes, ASP Conf. Ser., 101, 17

Avni, Y. 1976, ApJ, 210, 642

Beech, M. 1985, Ap\&SS, 117, 69

Bird, A. J., Coe, M. J., McBride, V. A., \& Udalski, A. 2012, MNRAS, 423, 3663

Campana, S., Stella, L., Israel, G. L., et al. 2002, ApJ, 580, 389

Charles, P. A., Booth, L., Densham, R. H., et al. 1983, MNRAS, 202, 657

Corbet, R. H. D., Charles, P. A., Southwell, K. A., \& Smale, A. P. 1997, ApJ, 476,833

Densham, R. H., Charles, P. A., Menzies, J. W., van der Klis, M., \& van Paradijs, J. 1983, MNRAS, 205, 1117

Doroshenko, V., Tsygankov, S. S., Mushtukov, A. A., et al. 2017, MNRAS, 466, 2143

Ducci, L., Covino, S., Doroshenko, V., et al. 2016, A\&A, 595, A103

Ferland, G. J., Chatzikos, M., Guzmán, F., et al. 2017, Rev. Mex. Astron. Astrofis., 53, 385

Hatchett, S., Buff, J., \& McCray, R. 1976, ApJ, 206, 847

Haubois, X., Carciofi, A. C., Rivinius, T., Okazaki, A. T., \& Bjorkman, J. E. 2012, ApJ, 756, 156

Hohle, M. M., Neuhäuser, R., \& Schutz, B. F. 2010, Astron. Nachr., 331, 349

Howarth, I. D., Prinja, R. K., Roche, P. F., \& Willis, A. J. 1984, MNRAS, 207, 287

Hynes, R. I. 2010, ArXiv e-prints [arXiv:1010.5770]

Kallman, T. R., \& McCray, R. 1982, ApJS, 50, 263

Klus, H., Ho, W. C. G., Coe, M. J., Corbet, R. H. D., \& Townsend, L. J. 2014, MNRAS, 437, 3863

Kretschmar, P., Wilms, J., Staubert, R., Kreykenbohm, I., \& Heindl, W. A 2004, 5th INTEGRAL Workshop on the INTEGRAL Universe, eds. V. Schoenfelder, G. Lichti, C. Winkler, ESA SP, 552, 329

Lee, U., Osaki, Y., \& Saio, H. 1991, MNRAS, 250, 432

Liu, Q. Z., van Paradijs, J., \& van den Heuvel, E. P. J. 2006, A\&A, 455, 1165

Maraschi, L., Treves, A., \& van den Heuvel, E. P. J. 1976, Nature, 259, 292

Maraschi, L., Traversini, R., \& Treves, A. 1983, MNRAS, 204, 1179

McGowan, K. E., \& Charles, P. A. 2003, MNRAS, 339, 748

Okazaki, A. T., \& Negueruela, I. 2001, A\&A, 377, 161

Pasinetti Fracassini, L. E., Pastori, L., Covino, S., \& Pozzi, A. 2001, A\&A, 367, 521

Ponman, T. J., Skinner, G. K., \& Bedford, D. K. 1984, MNRAS, 207, 621

Rajoelimanana, A. F., Charles, P. A., \& Udalski, A. 2011, MNRAS, 413, 1600

Rajoelimanana, A. F., Charles, P. A., Meintjes, P. J., et al. 2017, MNRAS, 464, 4133

Reig, P. 2011, Ap\&SS, 332, 1

Rivinius, T., Carciofi, A. C., \& Martayan, C. 2013, A\&ARv, 21, 69

Russell, S. C., \& Dopita, M. A. 1992, ApJ, 384, 508

Schmidtke, P. C., Cowley, A. P., \& Udalski, A. 2013, MNRAS, 431, 252

Skinner, G. K. 1980, Nature, 288, 141

Skinner, G. K. 1981, Space Sci. Rev., 30, 441

Skinner, G. K., Bedford, D. K., Elsner, R. F., et al. 1982, Nature, 297, 568

Tarenghi, M., Tanzi, E. G., Treves, A., et al. 1981, A\&AS, 43, 353

Tarter, C. B., Tucker, W. H., \& Salpeter, E. E. 1969, ApJ, 156, 943

Titarchuk, L. 1994, ApJ, 434, 570

Tjemkes, S. A., Zuiderwijk, E. J., \& van Paradijs, J. 1986, A\&A, 154, 77

Vrtilek, S. D., Raymond, J. C., Garcia, M. R., et al. 1990, A\&A, 235, 162

Waters, L. B. F. M., van den Heuvel, E. P. J., Taylor, A. R., Habets, G. M. H. J., \& Persi, P. 1988, A\&A, 198, 200

Waters, L. B. F. M., de Martino, D., Habets, G. M. H. J., \& Taylor, A. R. 1989, A\&A, 223, 207

White, N. E., \& Carpenter, G. F. 1978, MNRAS, 183, 11P

Zhukovska, S., \& Henning, T. 2013, A\&A, 555, A99 